\title{
Re-UCP Software Effort Estimation Method: A Critical Study
}

\author{
Mudasir Manzoor Kirmani \\ Research Scholar \\ School of CS \& IT, MANUU \\ Hyderabad, India
}

\author{
Syed Mohsin Saif \\ Research Scholar \\ School of CS \& IT, MANUU \\ Hyderabad, India
}

\author{
Abdul Wahid \\ Dean \& Head \\ School of CS \& IT, MANUU \\ Hyderabad, India
}

\begin{abstract}
Software Effort Estimation process is one the most important process in software project management. The importance of software in our daily lives is increasing with every passing day which has resulted in increase in the complexity of softwares. In order to manage software development projects with varying levels of complexity accurate software effort estimations play a pivotal role. This research work is an attempt to analyze the performance of Re-UCP method of software effort estimation based on the inputs from software industry and academia from India and abroad. This research work is an effort to find out the relevance of modifications made in Re-UCP model of software effort estimation from industrial perspective. This paper gives an insight into the responses received from consultants, costestimators, software engineers and researchers from academia after implementing Re-UCP effort estimation method in the their respective domains.
\end{abstract}

\section{Keywords}

Software Effort Estimation, Software effort validation, ReUCP, E-UCP, UCP, iUCP.

\section{INTRODUCTION}

With the swift expansion of software, people have much more reliance on computer based software systems for meeting their demands that occur on daily basis. Software systems are diverse in nature and play very important role not only in scientific and business enterprises, but also in day-to-day activities where it runs devices like cars, phones and other electronic gadget as well. With the advent of time the usage and applicability of computer based software systems have surged across vertical and horizontal domains at a rapid pace. At present most of the processes ranging from basic to complex, needs the services of different software systems directly or indirectly for effective and efficient completion. This has resulted in rapid increase in the demand of software development systems paving way to more opportunities for the software industry. Every software enterprise has to ensure the successful completion of a software system on time and within budget without compromising on quality of the software system to be developed. Software enterprises use different tools and techniques to ensure development of a software system on time and within budget however, to manage effectively the software development process different procedures are followed to monitor and manage the software development process from inception till maturity.

The most challenging issue that the software Industry encounters is less efficient management of software development budget projections. This problem has put the modern day software development companies in a situation wherein they are dealing with improper requirement engineering, ambiguous resource elicitation, uncertain cost and effort estimation which may lead to failure of the development process. The most inevitable aspect of a software development enterprise is to form a counter mechanism to deal with the problems which leads to chaos. An emphatic combative domain to deal with this problem is to schedule the whole development process to undergo proper and efficient estimation, where the estimation of all the resources can be made well in advance in order to check whether the conceived project is feasible and within the resources available. Software effort estimation is an activity to estimate how long it takes to accomplish a software development process or project. The effort estimation activity is very important to be able to know how much the relevant value of software is generated.

\section{RELATED WORK}

Most of the software development organizations are focusing on object oriented approach of software development at present. The basic building block of any objective oriented development is use-case diagram as the development framework is prepared using unified modelling language (UML). Use-case diagrams are prepared in early stages of software development after clearly understanding the user requirements and system constraints. The use-case diagram is the simplest representation of the user's interaction within the system. These diagrams can portray different types of users and their different interaction patterns. Use-case point is the unit of interaction between the system and users. More specifically use case diagram is composed of use cases and actors which represents the functional requirements whereas an actor is a role played by a user. Each use-case is represented by use case scenario diagram. The use case scenario is mainly composed of a success scenario and an alternative scenario. Researchers from academia as well as industry have shown interest in the Use Case Point based approaches because of the promising results obtained along with their early applicability in budget estimation. Some of the methods developed based on use case point based approached are UCP, iUCP, e-UCP and Re-UCP.

Use Case Point (UCP) model for software effort estimation was first developed by [4] based on the use case diagrams. The main aim of this model was to provide a simple estimation method which is easily adaptable to object oriented approach for developing software projects.

The iUCP model of software effort estimation provides an enhancement of UCP by incorporating HCI (human computer interface) concerns and claims to contribute as a consistent software effort estimation technique. iUCP is based on revised actor and use-case assessment criteria emerging from enhanced human-centric models of requirements such as the one proposed by the Wisdom method [16]. The rationale is that for interactive software system development early estimates based on models of requirements can be accurate only if they reflect the HCI concerns related to users and their interaction with the system [16]. 
The extended use-case point method (e-UCP) is another modified version of UCP method and was proposed by Kasi Perivasamy and Aditi Ghade in 2009 [17]. The e-UCP model considers some additional information about the relationships between actors and use-cases. The e-UCP is focussed on internal details of a use-case by including use-case narratives in effort estimation process of a software development project in the early stages of development [3] [5] [13] [17].

The revised use case point method (Re-UCP) was proposed by Kirmani and Wahid in 2015 [8]. The Re-UCP method of effort estimation was modified by revising the actor \& use-case classification and by including a new parameter in both technical and environmental complexity factor. The main aim of the Re-UCP model was to bridge the gap between approximated and actual software efforts in the rapidly changing software industry. The Re-UCP model was evaluated using different tools like MMRE, MdMRE and PRED and the results from the evaluation tools have been promising as reported by [7] [9] [12] [14] [15]. However, to validate the promising results of Re-UCP model this research work is an attempt to critically evaluate the performance of the software effort estimation model from the industrial perspective [17] [21].

\section{VALIDATION PROCESS}

The validation was planned to include review of the Re-UCP method of software effort estimation methods by different experts and practitioners from the software industry \& academia. The purpose of the validation process was to get the Re-UCP method examined by consultants, software professionals, software effort estimators and academic researchers. The validation process was primarily aimed at acquiring the practitioner's opinions on the use of Re-UCP method of software effort estimation. For this purpose a partially-open questionnaire was prepared as Review Form with specific questions to acquire data pertaining to the effectiveness, performance and impact of the changes made to use-case point based approaches of software effort estimation. The Review/validation format included the following questions:

i. How do you rate the impact of the following changes on software effort estimation?
a. Actor categorization in Re-UCP;
b. Use Case categorization and respective weight;
c. Addition of Scalability as T14 in TCF; and
d. Addition of Project Methodology as E9 in $\mathrm{ECF}$

ii. How do you rate the performance of Re-UCP in comparison with other use case point based software effort estimation methods?

iii. Can Re-UCP method of software effort estimation method be applied in early stages of software development?

iv. Overall rating of the Re-UCP method of effort estimation.

The respondents were requested to rate the questions based on a rating scale from 1-5 where rating 1- Not relevant; 2-Poor; 3Marginal; 4-Moderate; and 5-Good.

This questionnaire was administered over 110 identified experts from India and abroad through e-mail and personal interviews. It may not be wrong to say that even after making a considerable effort on acquiring the filled questionnaires; the researcher could gather duly filed forms, from 41 (forty one) experts India and abroad that are summarized in the proceeding sections. There is no doubt that the observations were found to be very valuable, and therefore have been examined in the following section.

The responses from different experts were analyzed and it was found that most of the experts were satisfied with the overall performance of the software effort estimation model. The graphical representations of responses received from the experts regarding validation of actor categorization in Re-UCP model is given in figure 1 where most of the responses have rated the actor categorization as good.

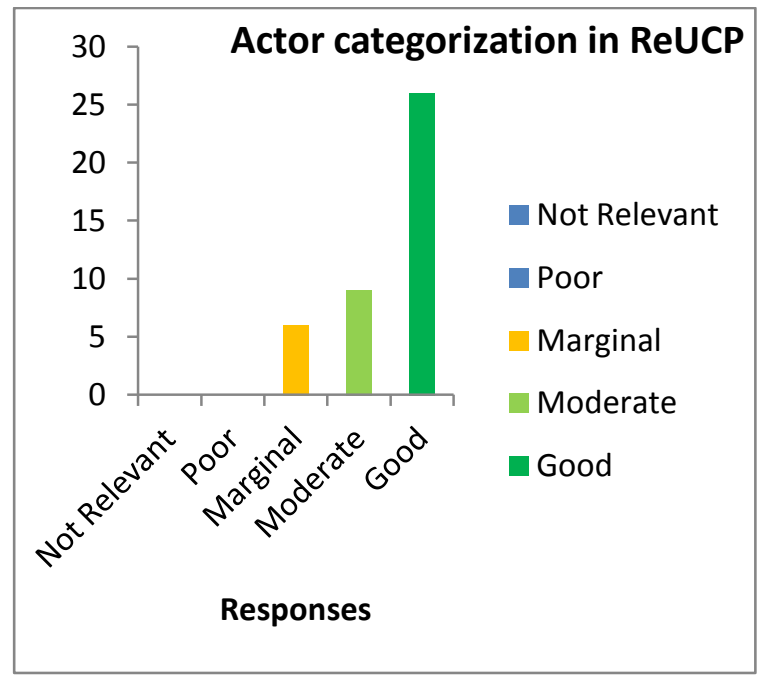

Fig 1: Impact of actor categorization in Re-UCP

The graphical representations of responses received from the experts regarding validation of use-case categorization and their respective weights in Re-UCP model is given in figure 2

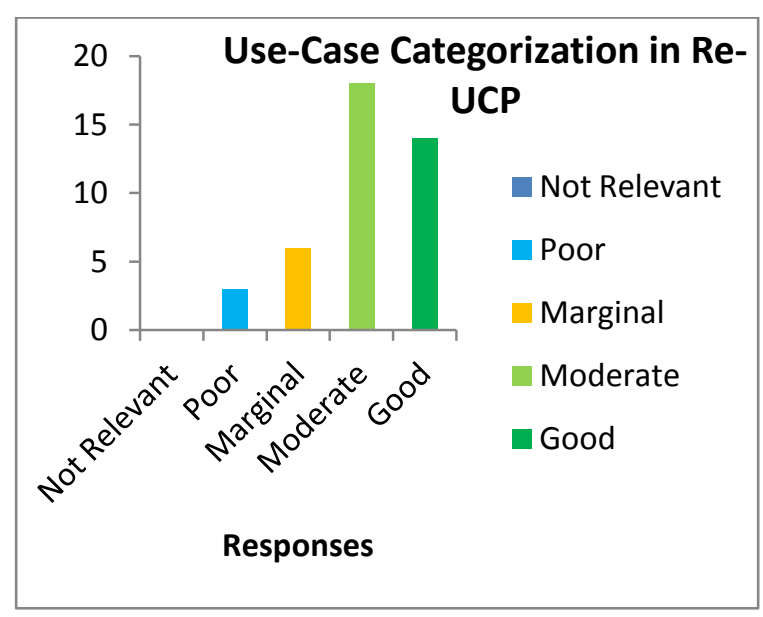

Fig 2: Impact of use-case categorization in Re-UCP

The graphical representations of responses received from the experts regarding addition of scalability as 14 th parameter in TCF and 09th parameter in ECF is shown in figure 3 and 4 respectively. 


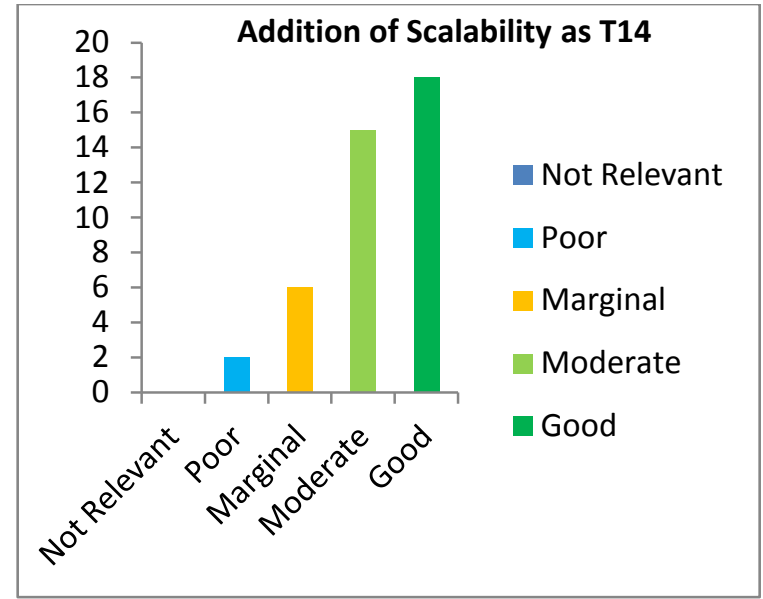

Fig 3: Impact of Scalability on estimated efforts using ReUCP

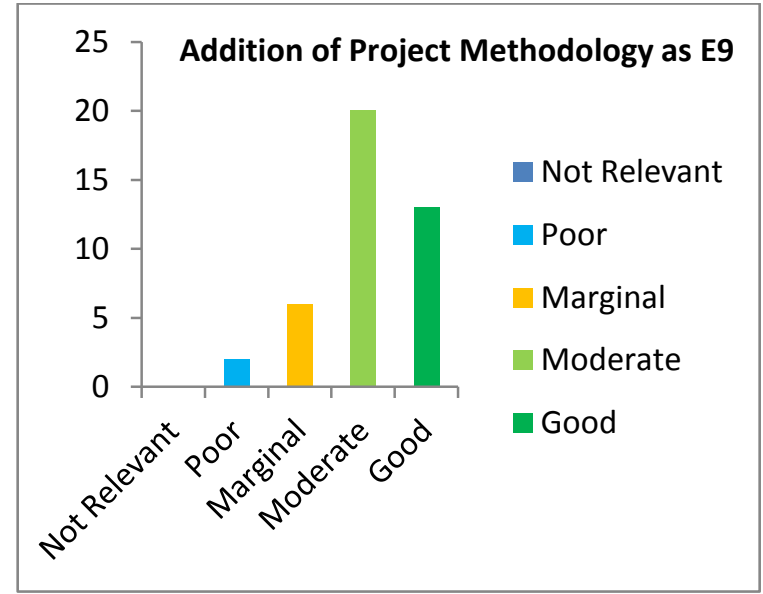

Fig 4: Impact of Project Methodology on estimated efforts using Re-UCP

Based on the responses received from the experts and researchers in the software effort estimation domain the performance of efforts estimated using Re-UCP in comparison with the other use-case point based methods is shown in figure 5. The summary of responses received for validating the relevance of Re-UCP method in estimating software efforts in early stages of development is shown in figure 6 .

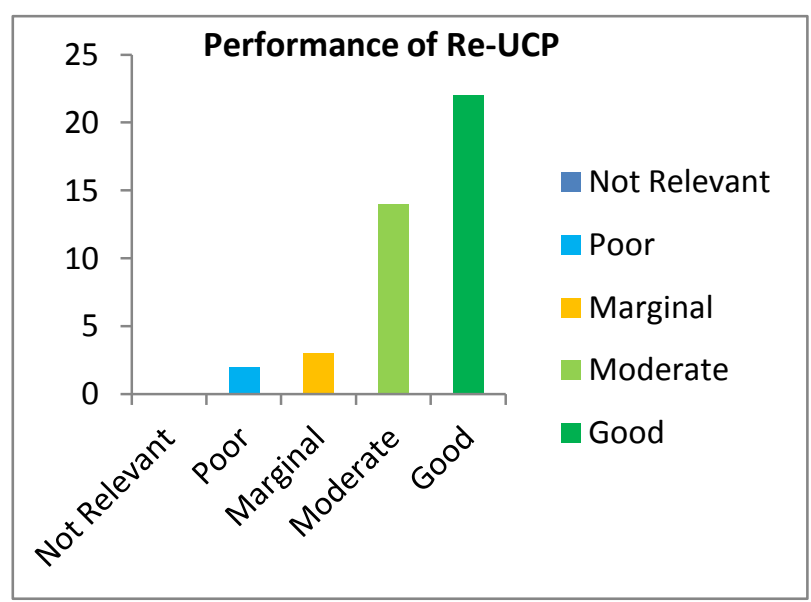

Fig 5: Performance of efforts estimated using Re-UCP

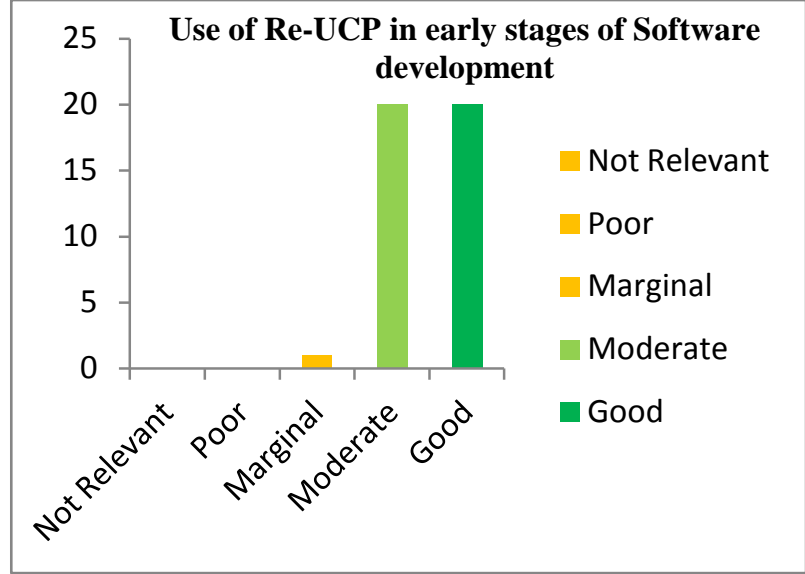

Fig 6: Relevance of Re-UCP method for estimating efforts in early stages of software development

The overall performance of Re-UCP method for estimation efforts of software development projects based on the responses received from the experts after validation are summarized in figure 7.

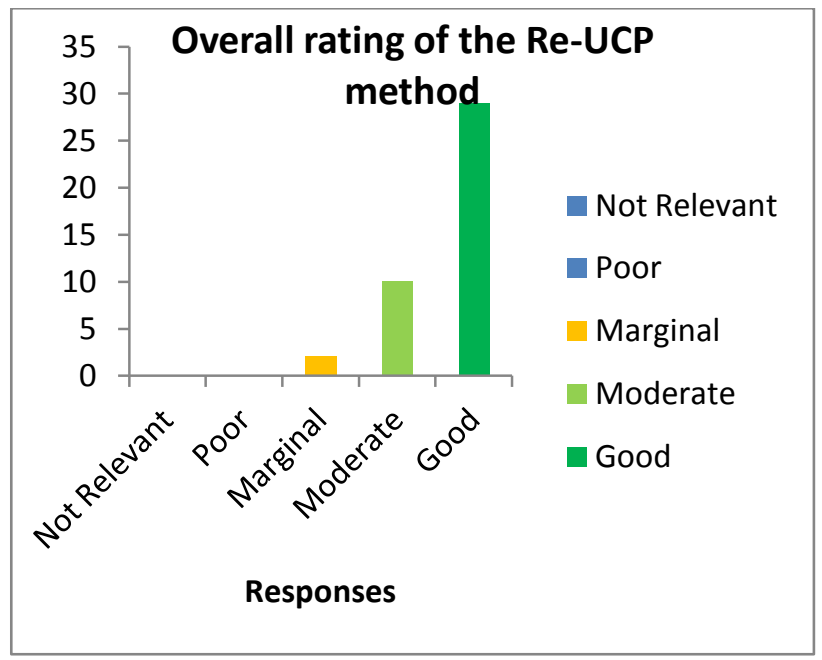

Fig 7: Overall performance of Re-UCP after validation.

The mean, standard deviation and variance of the responses received from the experts in software effort estimation domain is given in figure 8 and 9.

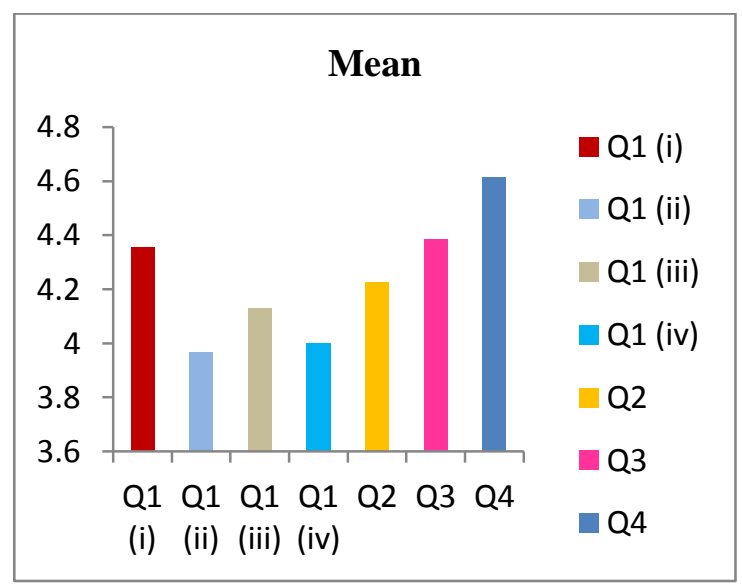

Figure 8: Mean of responses received in validation of ReUCP 


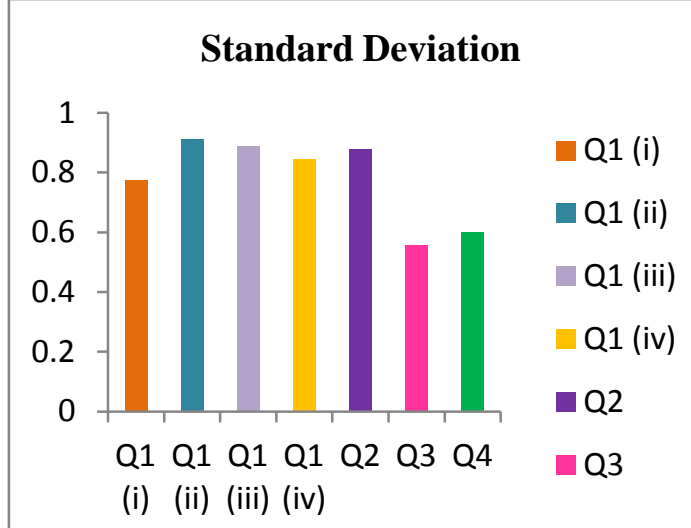

Fig 9: Standard Deviation of responses received in validation of Re-UCP

The percentage of means using 5-point scale regarding impact of modifications in Re-UCP is given in table 1.

Table 1: Percentage of Means for recommended changes in Re-UCP

\begin{tabular}{|c|l|c|c|c|}
\hline $\begin{array}{c}\text { S. } \\
\text { No }\end{array}$ & $\begin{array}{l}\text { Questions regarding } \\
\text { change }\end{array}$ & Mean & SD & $\begin{array}{c}\text { Percentage of } \\
\text { Mean }\end{array}$ \\
\hline 1 & $\begin{array}{l}\text { Actor categorization } \\
\text { in ReUCP }\end{array}$ & 4.35 & 0.798 & 87 \\
\hline 2 & $\begin{array}{l}\text { Use Case type and } \\
\text { respective weight }\end{array}$ & 3.97 & 0.948 & 79 \\
\hline 3 & $\begin{array}{l}\text { Addition of } \\
\text { Scalability as T14 }\end{array}$ & 4.13 & 0.922 & 83 \\
\hline 4 & $\begin{array}{l}\text { Addition of Project } \\
\text { Methodology as E9 }\end{array}$ & 4.00 & 0.856 & 80 \\
\hline
\end{tabular}

The percentage of means using 5-point scale for overall performance of Re-UCP is given in table 2 .

Table 2: Percentage of Means for performance of Re-UCP

\begin{tabular}{|c|c|c|c|c|}
\hline $\begin{array}{c}\text { S. } \\
\text { No. }\end{array}$ & $\begin{array}{c}\text { Questions regarding } \\
\text { change }\end{array}$ & Mean & SD & $\begin{array}{c}\text { Percentage } \\
\text { of Mean }\end{array}$ \\
\hline 1 & $\begin{array}{c}\text { Performance of Re- } \\
\text { UCP in comparison } \\
\text { with other use case } \\
\text { point methods. }\end{array}$ & 4.23 & 0.884 & 85 \\
\hline 2 & $\begin{array}{c}\text { Applicability in early } \\
\text { stages of development }\end{array}$ & 4.39 & 0.558 & 88 \\
\hline 3 & $\begin{array}{c}\text { Overall rating of Re- } \\
\text { UCP }\end{array}$ & 4.61 & 0.495 & 92 \\
\hline
\end{tabular}

\section{CONCLUSION}

The general comments of the reviewers suggest Re-UCP method of software effort estimation as a reliable and feasible model for estimating efforts required for software development projects. After analysing the expert comments, it is concluded that there is a general agreement among experts that the proposed method may be effectively used to estimate efforts of software projects.

This research work is just a platform for the research community to improve the existing models for benefit of the software industry in general and academia in particular. Keeping in mind rapid increase in complexity and data size in business enterprises the need of the hour is to use datasets from large and more complex software projects to validate the ReUCP model across different domains. Therefore, future research in this domain needs to be carried out to strengthen the approaches available for software effort estimation which will help the developers in reducing the gap between actual efforts and estimated efforts. The proposed model needs to be refined to develop an effort estimation model which will be tailor-made for estimation of efforts in web application development domain. This will not only give an easy to use method to the web application domain but will also help in approximating reliable estimates which are more closer to the actual efforts and the same will result in optimally managing the software development process.

\section{REFERENCES}

[1] Alves, R., Valente, P. \& Numes, N. J. (2013, June). Improving Software Effort Estimation with HumanCentric Models: a comparison of UCP and iUCP accuracy, In Proceedings of the 5th ACM SIGCHI symposium on Engineering interactive computing systems 287-296.

[2] Arnold, M. \& Pedross, P. (1998). Software size measurement and productivity rating in a large-scale software development department, Forging New Links, IEEE Computer Society, Los Alamitos, CA, 490-493.

[3] Jha, P., Jena, P. P. \& Malu, R. K. (2014). Estimating Software Development Effort using UML Use Case Point (UCP) Method with a Modified set of Environmental Factors, International Journal of Computer Science and Information Technologies (IJCSIT), 5(3), 2742-2744.

[4] Karner, G. (1993). Metrics for objectory. Sweden: University of Linköping. Sweden. No. LiTHIDA- Ex9344,21.

[5] Kashyap, D., Shukla, D. \& Misra, A. K. (2014). Refining the Use Case Classification for Use Case Point Method for Software Effort Estimation, Proceedings of International Conference on Recent Trends in Information, Telecommunication and Computing, ACEEE Association of Computer Electronics and Electrical Engineers.

[6] Kemerer, C. F. (1987). An Empirical Validation of Software Cost Estimation Models. Communications of the ACM, 30(5), 416-429.

[7] Kirmani, M.M. and Wahid, A., (2015), "Impact of Modifications made in Re-UCP on software effort estimation" Journal of software engineering and applications, Vol. 8, No. 6, pp276-289

[8] Kirmani, M.M. and Wahid, A., (2015), "Revised Use Case Point (Re-UCP) Model for Software Effort Estimation" International Journal of Advanced Computer Science and Applications (IJACSA), Vol. 6, No. 3, pp65-71. U.S ISSN : 2156-5570(Online), U.S ISSN : 2158-107X

[9] Kirmani, M.M. and Wahid, A., (2015), "Use Case Point and e-Use Case Point method of software effort estimation: A critical performance comparison" International Journal of Computer Application Vol. 5, No. 3, pp55-64.

[10] Kirmani, M.M. and Wahid, A., (2015), "Use Case Point Method of Software Effort Estimation: A Review" International Journal of Computer Applications Vol. 116, No.15, pp43-47, April 2015. ISSN: 0975-8887

[11] Nageswaran, S. (2001). Test effort estimation using use case points. In Quality Week, 1-6.

[12] Nassif, A. B. (2010). Enhancing Use Case Points Estimation Method using Soft Computing Techniques, 
Journal of Global Research in Computer Science, 1(4), 1221.

[13] Nassif, A. B., (2012).Software Size and Effort Estimation from Use Case Diagrams Using Regression and Soft Computing Models, PhD thesis, Western University Ontario. (available

http://ir.lib.uwo.ca/cgi/viewcontent.cgi?article $=1732 \&$ context $=$ etd ).

[14] Nassif, A. B., Capretz, L. F., Ho, D. \& Azzeh, M. (2012). A treeboost model for software effort estimation based on use case points, (ICMLA) 11th International Conference on Machine Learning and Applications IEEE, 2, 314-319.

[15] Nassif, A. B., Ho, D. \& Capretz, L. F. (2011). Regression model for software effort estimation based on the use case point method, International Conference on Computer and Software Modelling, 14, 106-110.

[16] Nunes, N. J. (2010). iUCP - estimating interaction design projects with enhanced use case points. Proceedings of the 8th international conference on Task Models and Diagrams for User Interface Design, Springer, 131-145.
[17] Periyasamy, K. \& Ghode, A. (2009). Cost Estimation Using Extended Use Case Point (e-UCP) Model, International Conference on Computational Intelligence and Software Engineering.

[18] Robiolo, G., Badano, C., Orosco, R., (2009, October) Transactions and paths: two use case based metrics which improve the early effort estimation, Third International Symposium on Empirical Software Engineering and Measurement, ESEM 2009. 3rd International Symposium, 422-425.

[19] Schneider, G. \&Winters, J. P. (2001). Applied use Cases, Second Edition, A Practical Guide. Addison-Wesley.

[20] Sholiq, Sutanto T., Widodo A. P., \& Kurniawan W. (2014). Effort rate on use case point methods for efforts estimation of website of development, Journal of Theoretical and Applied Information Technology, 63(1), $209-218$.

[21] Wang, F., Yang, X., Zhu X. \& Chen, L.(2009, December) "Extended use case points method for software cost estimation," International Conference on Computational Intelligence and Software Engineering, IEEE, 1-5. 